\title{
Dissecting the bone marrow HSC niches
}

\author{
Cell Research (2016) 26:975-976. doi:10.1038/cr.2016.71; published online 14 June 2016
}

\begin{abstract}
Hematopoietic stem cells (HSCs) sponsoring hematopoiesis are preserved in the bone marrow (BM) microenvironment or niche. Here, Itkin et al. demonstrate how distinct blood vessels contribute to HSC maintenance and hematopoiesis in the $\mathrm{BM}$ niches.
\end{abstract}

Hematopoiesis is maintained by HSCs, which have extensive selfrenewal capacity and multipotent differentiation potential to reconstitute all hematopoietic lineages. The BM is a complex organ with medullary cavity surrounded by a shell of bone, including cancellous bone or trabecular bone, normally at the ends of long bone and cortical bone, the majority of long bone. Small projections of bone are found throughout the trabecular bone, where vascular structures are enriched in the inner bone surface termed "endosteum". The BM is known to be the primary site for HSC maintenance and hematopoiesis. The HSC niche conceptually proposed by Ray Scofield in 1978 was first functionally identified in the endosteal region of trabecular bone, termed the "endosteal niche" $[1,2]$, which is now known to be constructed of multiple cell components rather than single type osteoblastic cells. However, later studies revealed that a large portion of HSCs are localized by the venous sinusoids in the central marrow, thus the "vascular niche" was identified. Subsequent controversial reports and the debate whether endosteal niche or vascular niche is the "real" HSC niche largely stemmed from directly applying a previous paradigm of the Drosophila germ stem cell niche comprising a single site with a single type of niche cells maintaining a homogeneous stem cell population [3] to the adult BM system. Actually, in adult BM, multiple niche cell populations from distinct $\mathrm{BM}$ zones have been reported to regulate HSCs for long-term maintenance, proliferation, and differentiation. A recent report by Itkin et al. [4] provided strong evidence to support the concept that different coexisting niche compartments regulate HSCs in different states.

HSC maintenance is associated with quiescence and low metabolic state to protect genomic integrity and to retain functional capacity. However, to support hematopoiesis, HSCs need to be activated to proliferate and to replenish lost or damaged blood cells. Although the regulation of these two different HSC states - quiescent vs active - is not fully understood, a growing body of evidence indicates that HSCs in different stages are maintained in distinct BM zones. Murine BM is highly vascularized with large arteries, mainly in central marrow, branching into small arterioles that transit into venous sinusoids, either in the central marrow or near the endosteum proximal to trabecular bone, connecting with veins and eventually with central veins that carry blood out of the marrow. Homed long-term repopulating HSCs are often detected in the endosteal niche [5]. In addition, quiescent HSCs are also maintained by arterioles rather than sinusoids in the BM [6]. Later studies further showed that blood vessels in separate zones are associated with different surrounding cells, such as endosteal bone lining cells, neuronal cells, mesenchymal stem/progenitor cells, or megakaryocytes, which independently contribute to HSC maintenance [7-10]. Therefore, these different intersection sites between blood vessels and their surrounding cells provide distinct niches for HSC maintenance and regulation. Clearly, distinct niches exist in the BM for HSC regulation; however, their cellular composition and unique feature for HSC regulation have not yet been fully explored.

In the recent report, Itkin et al. [4] analyzed blood vessels located at different BM zones. Sinusoids, arteries, and endosteal small arterioles are actually composed of different types of endothelial cells, which can be separated based on molecular markers. Besides CD31 as a general endothelial cells marker, both arterial endothelial cells and endosteal small arteriole endothelial cells are positive for Sca-1 and Nestin, but sinusoid endothelial cells are not. Arteries and small arterioles can be further distinguished by morphology and a molecular marker, Endomucin, which is negative for arteries, but positive for small arterioles in the endosteal region [11]. Those different endothelial cells are influenced by different types of surrounding cells, including pericytes and endosteal bone lining cells. Arterial endothelial cells are surrounded by $\alpha \mathrm{SMA}^{+}$cells, and small endosteal arterioles are surrounded by $\mathrm{Nestin}^{+}$and PDGFR $\alpha^{+}$mesenchymal stem and progenitor cells proximal to trabecular bone. Although sinusoids comprise a single layer of endothelial cells, megakaryocytes are often associated with particular sinusoid sites. After sketching the cellular contours of BM zones, Itkin et al. further investigated how these distinct zones contribute to HSC regulation. They first discovered 


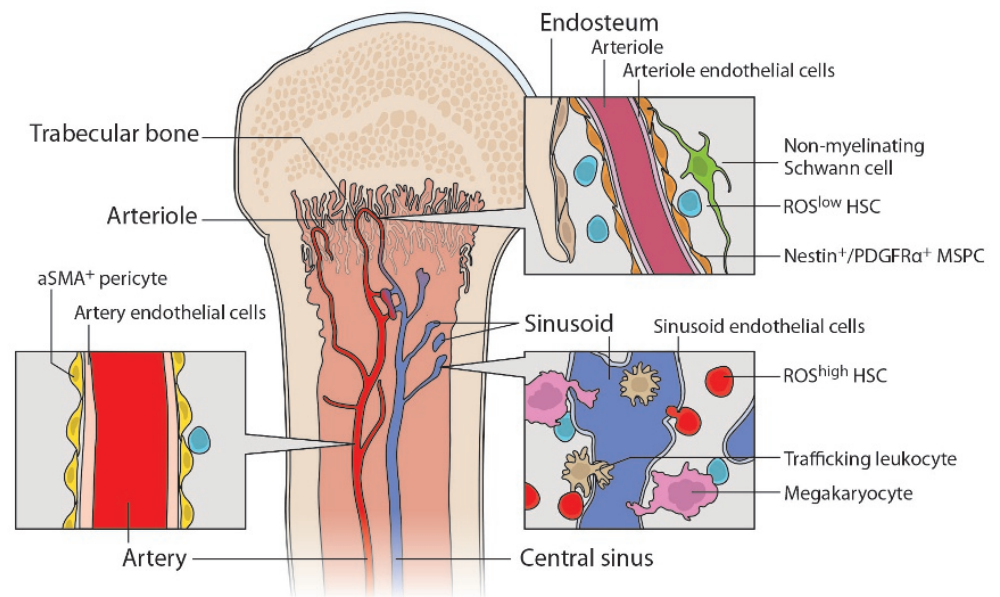

Figure $1 \mathrm{HSCs}$ in different states are maintained in distinct BM zones. In each zone, blood vessels are constructed by different types of endothelial cells and surrounded by their unique perivascular cells. ROS ${ }^{\text {low }}$ quiescent HSCs are maintained by arteries, endosteal arterioles, and certain sinusoid sites by megakaryocytes. ROS ${ }^{\text {high }}$ active HSCs are kept by sinusoids where leukocyte and HSC trafficking is happening.

the distribution of HSCs with different metabolic activities reflected by their reactive oxygen species (ROS) levels. They found that ROS ${ }^{\text {low }}$ HSCs, indicating lower metabolic state and thus quiescent, are localized mainly adjacent to arterioles and small arterioles proximal to endosteal zones. However, ROS ${ }^{\text {high }}$ HSCs, indicating higher metabolic state and thus active, with accelerated differentiation and elevated risk of exhaustion, are localized by sinusoids in the central marrow (Figure 1).

More interestingly, Itkin et al. revealed with in vivo live imaging that sinusoids are much more permeabilized than arteries and small arterioles, which suggests that sinusoids are an exclusive site for leukocyte (and HSC) tracking from the $\mathrm{BM}$ to circulation. Moreover, Itkin et al. showed that Fibroblast growth factor signaling plays an important role in maintaining endothelial integrity which keeps HSCs from blood plasma exposure. Exposing HSCs to peripheral blood plasma increased their ROS levels, which augments their differentiation but compromises their long-term repopulating potential. This study demonstrated that the fenestrated sinusoid endothelium activates HSCs and increases their differentiation via penetrating the peripheral blood plasma. Itkin et al. also noticed that in certain sinusoid sites, HSCs being adjacent to megakaryocytes kept them in a ROS ${ }^{\text {low }}$ quiescent state. This observation is consistent with previous reports in which megakaryocytes maintain HSC quiescence via TGF $\beta$ and CXCL4 signalings $[9,10]$. Thus HSCs are temporarily protected in this megakaryocyte-sinusoid zone to restrict their proliferation and differentiation. However, further studies are needed to explore whether and how the other pericytes, such as smooth muscle cells, mesenchymal stem and progenitor cells, neuronal cells, and endosteal bone lining cells, interact with less penetrated arterials/endosteal small arterioles and harmoniously contribute to HSC maintenance.

Itkin et al. also provide insight into improving clinical BM transplantation. Clinical protocols to mobilize HSCs from the BM to the circulation have been widely used in stem cell transplantation. However, based on results from this study, exposure to peripheral blood plasma leads to an increased ROS level in HSCs, which can significantly compromise their long-term self-renewal potential [12]. Therefore, limiting HSC loitering in peripheral blood or using the ROS scavenger N-acetyl-L-cysteine (NAC) to protect mobilized HSCs should be considered to improve current protocols. Furthermore, how to recover the endothelial barrier following stem cell transplants should be considered since radiation injury of blood vessels is a common clinical problem which may lead to enhanced plasma penetration and compromised stem cell function. Thus, the study results suggest that restricting donor HSC exposure to peripheral plasma and facilitating recovery of recipient blood vessel integrity post myeloablation may augment current clinical BM transplant efficiency.

\section{Meng Zhao ${ }^{1,2}$, Linheng $\mathrm{Li}^{1,3}$}

${ }^{1}$ Stowers Institute for Medical Research, Kansas City, MO 64110, USA; ${ }^{2}$ Center for Stem Cell Biology and Tissue Engineering, Department of Pathophysiology, Zhongshan Medical School, Sun Yat-Sen University, Guangzhou, Guangdong 510080, China; ${ }^{3}$ Department of Pathology and Laboratory Medicine, University of Kansas Medical Center, Kansas City, KS 66160, USA Correspondence: Linheng $\mathrm{Li}$

E-mail: LIL@stowers.org

\section{References}

1 Zhang J, Niu C, Ye L, et al. Nature 2003; 425:836-841.

2 Calvi LM, Adams GB, Weibrecht KW, et al. Nature 2003; 425:841-846.

3 Xie T, Spradling AC. Science 2000; 290:328-330.

4 Itkin T, Gur-Cohen S, Spencer JA, et al. Nature 2016; 532:323-328.

5 Xie Y, Yin T, Wiegraebe W, et al. Nature 2009; 457:97-101.

6 Kunisaki Y, Bruns I, Scheiermann C, et al. Nature 2013; 502:637-643.

7 Yamazaki S, Ema H, Karlsson G, et al. Cell 2011; 147:1146-1158.

8 Mendez-Ferrer S, Michurina TV, Ferraro F, et al. Nature 2010; 466:829-834.

9 Zhao M, Perry JM, Marshall H, et al. Nat Med 2014; 20:1321-1326.

10 Bruns I, Lucas D, Pinho S, et al. Nat Med 2014; 20:1315-1320.

11 Kusumbe AP, Ramasamy SK, Itkin T, et al. Nature 2016; 532:380-384.

12 Mantel CR, O'Leary HA, Chitteti BR, et al. Cell 2015; 161:1553-1565. 